\title{
The inactivation of the basolateral nucleus of the rat amygdala has an anxiolytic effect in the elevated T-maze and light/dark transition tests
}

C.H. Bueno ${ }^{1}$, H. Zangrossi Jr. ${ }^{2}$ and M.B. Viana ${ }^{3}$

\author{
${ }^{1}$ Laboratório de Psicofarmacologia, Faculdade de Filosofia, \\ Ciências e Letras de Ribeirão Preto, ${ }^{2}$ Departamento de Farmacologia, \\ Faculdade de Medicina de Ribeirão Preto, Universidade de São Paulo, \\ Ribeirão Preto, SP, Brasil \\ ${ }^{3}$ Departamento de Psicologia do Desenvolvimento, Faculdade de Psicologia, \\ Pontifícia Universidade Católica, São Paulo, SP, Brasil
}

Correspondence

M.B. Viana

Departamento de Psicologia do

Desenvolvimento

Faculdade de Psicologia, PUC

05014-901 São Paulo, SP

Brasil

E-mail: milena@usp.br

Research supported by FAPESP and CNPq.

Received March 22, 2005 Accepted August 9, 2005

\begin{abstract}
Pharmacological evidence indicates that the basolateral nucleus of the amygdala (BLA) is involved in the mediation of inhibitory avoidance but not of escape behavior in the elevated T-maze test. These defensive responses have been associated with generalized anxiety disorder (GAD) and panic disorder, respectively. In the present study, we determined whether the BLA plays a differential role in the control of inhibitory avoidance and escape responses in the elevated T-maze. Male Wistar rats (250-280 g, N = 9-10 in each treatment group) were pre-exposed to one of the open arms of the maze for $30 \mathrm{~min}$ and $24 \mathrm{~h}$ later tested in the model after inactivation of the BLA by a local injection of the $\mathrm{GABA}_{\mathrm{A}}$ receptor agonist muscimol $(8 \mathrm{nmol}$ in $0.2 \mu \mathrm{L})$. It has been shown that a prior forced exposure to one of the open arms of the maze, by shortening latencies to withdrawal from the open arm during the test, improves the escape task as a behavioral index of panic. The effects of muscimol in the elevated T-maze were compared to those caused by this GABA agonist in the avoidance reaction generated in the light/dark transition test. This defensive behavior has also been associated with GAD. In the elevated T-maze, intra-BLA injection of muscimol impaired inhibitory avoidance (control: 187.70 \pm 14.90 s, muscimol: $37.10 \pm 2.63 \mathrm{~s}$ ), indicating an anxiolytic effect, without interfering with escape performance. The drug also showed an anxiolytic effect in the light/dark transition test as indicated by the increase in the time spent in the lighted compartment (control: 23.50 $\pm 2.45 \mathrm{~s}$, muscimol: $47.30 \pm 4.48 \mathrm{~s}$ ). The present findings point to involvement of the BLA in the modulation of defensive responses that have been associated with GAD.
\end{abstract}

Key words

- Muscimol

- Generalized anxiety

disorder

- Panic disorder

- Amygdala

- Elevated T-maze

- Light/dark transition test in the rat 
The amygdala complex has been implicated in fear and anxiety (1). There is evidence that this limbic area is critical for the anxiolytic effect of benzodiazepine drugs (2). $\mathrm{GABA}_{\mathrm{A}} /$ benzodiazepine receptors are found in different nuclei of the amygdaloid complex, with the highest concentration present in the basolateral nuclear complex (3).

Administration of benzodiazepines or $\mathrm{GABA}_{\mathrm{A}}$ receptor agonists into the basolateral nucleus of the amygdala (BLA) causes anxiolytic effects in different animal models of anxiety, such as the elevated plus-maze $(4,5)$, the social interaction test $(6)$, the passive avoidance model (7), and the contextual fear conditioning test (8).

Evidence obtained in our laboratory indicates that the BLA may play a differential role in the regulation of defensive behaviors that have been associated with distinct subtypes of anxiety disorders. More specifically, we observed that microinjection of the benzodiazepine receptor agonist midazolam into this nucleus impaired the acquisition of inhibitory avoidance in the elevated T-maze without interfering with the expression of escape behavior in the same test (9).

In the elevated T-maze, inhibitory avoidance and escape responses are measured in a maze consisting of three elevated arms - one of them enclosed and two open. For inhibitory avoidance measurement, rats are placed at the distal end of the enclosed arm and the latency to withdraw from this arm with the four paws is recorded in three successive trials. Because of their innate fear of height and openness (10), rats learn to remain longer in the enclosed arm over trials, indicating the acquisition of inhibitory avoidance to the open arms. On the other hand, when the animals are placed at the end of one of the open arms they move towards the enclosed arm, presumably performing an escape response. Based on the effects of different classes of anxiety-modulating drugs in this test, inhibitory avoidance has been related to generalized anxiety disorder (GAD) and es- cape to panic disorder (11-16). The results of the study with midazolam in the elevated Tmaze indicate that the BLA exerts preferential control on defensive behaviors that have been associated with GAD, but not with panic disorder (9). Nevertheless, given the elevated $\mathrm{T}$-maze test protocol employed by the authors, the inefficacy of this drug regarding the elevated T-maze escape performance may be a false-negative result. In the mentioned analysis, inhibitory avoidance and escape were measured in rats that had no previous experience with the open arms of the elevated T-maze. It has been shown that a prior forced exposure to one of the open arms of the maze, by shortening latencies to withdrawal from the open arm during the test, improves the escape task as a behavioral index of panic $(16,17)$. For instance, whereas in naive rats chronic treatment with the antidepressant compound imipramine, which has also been used in clinical settings to treat panic disorder, did not affect escape, in rats previously exposed to the open arm the drug inhibited this behavior, indicating a panicolytic-like effect (17). Thus, more recent studies using the elevated T-maze have incorporated this methodological modification (for a review, see Refs. 17-19).

In light of these considerations, in the present study we further addressed the involvement of the BLA in the regulation of the two defensive behaviors measured by the elevated T-maze. To this end, we investigated the effect of the inactivation of this nucleus by means of local injection of the $\mathrm{GABA}_{\mathrm{A}}$ receptor agonist muscimol in rats previously exposed to one of the elevated $\mathrm{T}$ maze open arms. The effects of muscimol in this test were compared to those obtained in the light/dark transition model, an animal model that has been related to GAD (15).

Male Wistar rats weighing 250-280 g were stereotaxically implanted with stainless-steel guide cannulae aimed at the BLA. Six or seven days after surgery, the animals were tested in the elevated T-maze and light/ 
dark transition model. The elevated T-maze consists of three arms of equal dimensions $(50 \mathrm{~cm} \mathrm{x} 12 \mathrm{~cm}$ ) elevated $50 \mathrm{~cm}$ from the floor. One arm, enclosed by $40-\mathrm{cm}$ high walls, was perpendicular to two opposed open arms. One day before testing, the animals were exposed to one of the open arms for $30 \mathrm{~min}$ as described by Sena et al. (20). The light/dark test apparatus was a wood box $(48 \times 24 \times 27 \mathrm{~cm})$ divided by a barrier possessing a doorway $(10 \times 10 \mathrm{~cm})$ through which rats could pass into two chambers of equal measures: one painted black, not illuminated, and one painted white and brightly illuminated by a 60 -lux light source. On the test day, rats were injected bilaterally $(0.2$ $\mu \mathrm{L}, 2 \mathrm{~min}$ ) with muscimol ( $8 \mathrm{nmol}, \mathrm{N}=9$ ) or saline $(\mathrm{N}=10)$. Ten minutes later, inhibitory avoidance was measured by recording the time taken by the rats to withdraw from the enclosed arm of the elevated T-maze in three consecutive trials at 30-s intervals (baseline, avoidance 1 and avoidance 2 latencies). Following avoidance training (30 s), each animal was placed at the end of the same open arm as used in the pre-exposure session and the time taken to leave this arm was recorded in three consecutive trials (escape 1 to 3 ), again with 30 -s inter-trial intervals. Immediately after the tests in the elevated T-maze, the animals were tested in the light/dark transition model. Each rat was placed in the middle of the lighted compartment facing the doorway separating the two compartments. After the first transition from the lighted to the dark compartment, the behavior of the animals was recorded for an additional 5-min period for measurements of two parameters: the total number of transitions between the two compartments, and the time spent in the lighted compartment. For further details of both tests, see Sena et al. (20). After the behavioral tests, the animals were killed and their brains removed to examine the location of the injection site. Only data from animals injected into the BLA were considered for statistical analysis.
Figure 1A shows that intra-BLA injection of muscimol impaired inhibitory avoidance acquisition, indicating an anxiolytic effect. Repeated measure ANOVA showed a significant effect of trials $(\mathrm{F}(2,36)=111.55$; $\mathrm{P}<0.01)$, treatment $(\mathrm{F}(1,18)=156.61 ; \mathrm{P}<$ $0.01)$ and treatment by trial interaction $(\mathrm{F}(2,36)=65.73 ; \mathrm{P}>0.01)$. The unpaired Student $t$-test showed that animals treated with muscimol showed significantly $(\mathrm{P}<$ 0.01 ) shorter avoidance 1 and 2 latencies compared to control.

On the other hand, escape was not altered by intra-BLA injection of muscimol (Figure 1B). Repeated measure ANOVA showed an effect of trials $(\mathrm{F}(2,36)=45.69 ; \mathrm{P}<0.01)$, but not of treatment $(\mathrm{F}(1,18)=0.05 ; \mathrm{P}>$ $0.05)$ and no interaction between treatment and trials $(\mathrm{F}(1,18)=0.49 ; \mathrm{P}>0.05)$.

Figure 2A shows that in the light/dark transition model, intra-BLA injection of muscimol increased the time spent in the lighted compartment (Student $t$-test: $\mathrm{t}(18)=$ -5.14; $\mathrm{P}<0.01$ ), indicating an anxiolytic effect. The drug did not significantly affect

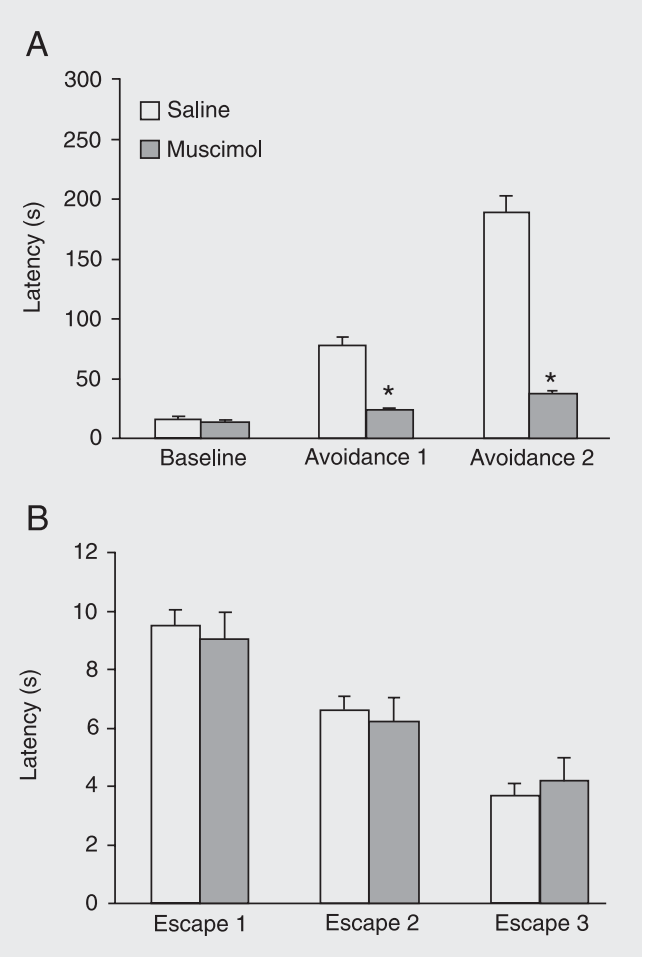

Figure 1. Effect of intra-basolateral nucleus of the amygdala injection of muscimol or saline on inhibitory avoidance $(A)$ and escape (B) latencies measured in the elevated T-maze. Twentyfour hours before the test, all animals were exposed to one of the open arms for $30 \mathrm{~min}$. The animals received $8 \mathrm{nmol} / 0.2 \mu \mathrm{L}$ of the drug. Data are reported as means \pm SEM for $\mathrm{N}=9-10$ animals. ${ }^{*} P<0.01$ compared with the saline group in the same trial (unpaired Student $t$-test). 
the number of transitions between the two compartments.

Therefore, the present results showed that the inactivation of the BLA by local microinjection of muscimol caused anxiolytic effects in two different animal models of GAD.

The effect of muscimol on the elevated T-maze and light/dark transition models does

Figure 2. Effect of intra-basolateral nucleus of the amygdala injection of muscimol or saline on the time spent in the lighted compartment $(A)$ and the number of transitions between compartments (B) in the light/dark transition test. The animals received 8 $\mathrm{nmol} / 0.2 \mu \mathrm{L}$ of the drug. Data are reported as means \pm SEM for $\mathrm{N}=9-10$ animals. ${ }^{*} \mathrm{P}<0.01$ compared with the saline group (unpaired Student $t$-test).

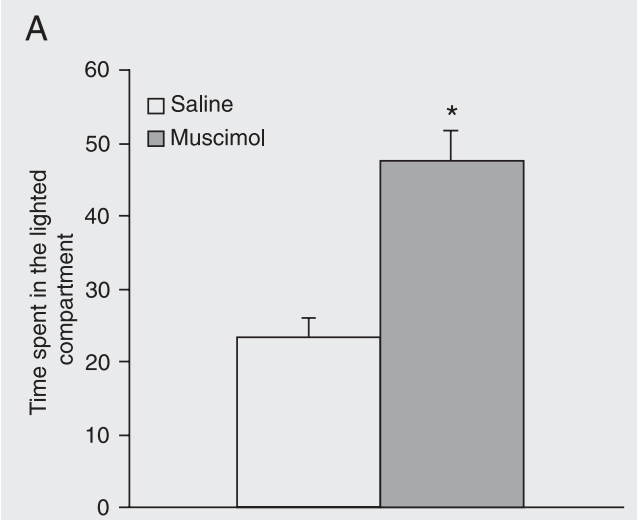

B

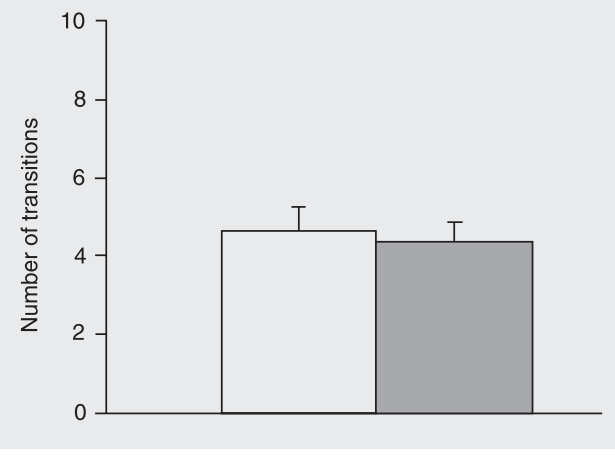

not seem to be related to a primary effect of this compound on the locomotor activity of the animals. In this respect, although the motor demand for inhibitory avoidance and escape in the elevated T-maze is nearly the same, muscimol shortened avoidance latencies without interfering with escape latencies. In the light/dark test, the drug enhanced the time spent in the lighted compartment without affecting the number of transitions between the two compartments. Changes in the latter parameter have also been used to infer the effect of a given drug on motor performance (for a review, see Ref. 15).

The present findings agree with the results of a study showing that intra-BLA/ lateral injection of the benzodiazepine receptor agonist midazolam impaired inhibitory avoidance acquisition, but did not change escape behavior in the elevated T-maze (9). In contrast to the present study, in the previous study rats were tested in the elevated $\mathrm{T}$ maze without prior experience in the open arms. Therefore, the present study suggests that, independently of the test protocol used, activation of $\mathrm{GABA}_{\mathrm{A}}$ /benzodiazepine receptors in the BLA selectively interferes with the inhibitory task in the elevated T-maze.

Overall, the results reported herein provide further evidence for the involvement of the BLA in the modulation of defensive responses that have been associated with GAD.

\section{References}

1. LeDoux J (2003). The emotional brain, fear, and the amygdala. Cellular and Molecular Neurobiology, 23: 727-738.

2. Nagy J, Zambo K \& Decsi L (1979). Anti-anxiety action of diazepam after intra-amygdaloid application in the rat. Neuropharmacology, 18: $573-576$

3. Niehoff DL \& Kuhar MJ (1983). Benzodiazepine receptors: localization in rat amygdala. Journal of Neuroscience, 3: 2091-2097.

4. Green S \& Vale AL (1992). Role of amygdaloid nuclei in the anxiolytic effects of benzodiazepines in rats. Behavioural Pharmacology, 3: $261-264$.

5. Zangrossi Jr H \& Graeff FG (1994). Behavioral effects of intraamygdala injections of GABA and 5-HT acting drugs in the elevated plus-maze. Brazilian Journal of Medical and Biological Research,
27: 2453-2456

6. Gonzalez LE, Andrews N \& File SE (1996). 5-HT 1 A and benzodiazepine receptors in the basolateral amygdala modulate anxiety in the social interaction test, but not in the elevated plus-maze. Brain Research, 732: 145-153.

7. Harris JA \& Westbrook RF (1995). Effects of benzodiazepine microinjection into the amygdala or periaqueductal gray on the expression of conditioned fear and hypoalgesia in rats. Behavioral Neuroscience, 109: 295-304.

8. Helmstetter FJ \& Bellgowan PS (1994). Effects of muscimol applied to the basolateral amygdala on acquisition and expression of contextual fear conditioning in rats. Behavioral Neuroscience, 108: 1005-1009. 
9. Zangrossi Jr H, Viana MB \& Graeff FG (1999). Anxiolytic effect of intra-amygdala injection of midazolam and 8-hydroxy-2-(di-npropylamino)tetralin in the elevated T-maze. European Journal of Pharmacology, 369: 267-270.

10. Treit D, Menard J \& Royan C (1993). Anxiogenic stimuli in the elevated plus-maze. Pharmacology, Biochemistry, and Behavior, 44: 463-469.

11. Graeff FG (1993). Role of 5-HT in defensive behavior and anxiety. Reviews in the Neurosciences, 4: 181-211.

12. Viana MB, Tomaz C \& Graeff FG (1994). The elevated T-maze: a new animal model of anxiety and memory. Pharmacology, Biochemistry, and Behavior, 49: 549-554.

13. Zangrossi Jr H \& Graeff FG (1997). Behavioral validation of the elevated T-maze, a new animal model of anxiety. Brain Research Bulletin, 44: 1-5.

14. Graeff FG, Netto CF \& Zangrossi Jr H (1998). The elevated T-maze as an experimental model of anxiety. Neuroscience and Biobehavioral Reviews, 23: 237-246.
15. Graeff FG \& Zangrossi Jr H (2002). Animal models of anxiety. In: D'Haenen H, Boer JA \& Willner P (Editors), Biological Psychiatric. John Wiley \& Sons Ltd., London, England, 879-893.

16. Poltronieri SC, Zangrossi Jr H \& Viana MB (2003). Antipanic-like effect of serotonin reuptake inhibitors in the elevated T-maze. Behavioural Brain Research, 147: 185-192.

17. Teixeira RC, Zangrossi Jr H \& Graeff FG (2000). Behavioral effects of acute and chronic imipramine in the elevated T-maze model of anxiety. Pharmacology, Biochemistry, and Behavior, 65: 571-576.

18. Graeff FG (2002). On serotonin and experimental anxiety. Psychopharmacology, 163: 467-476.

19. Graeff FG (2004). Serotonin, the periaqueductal gray and panic. Neuroscience and Biobehavioral Reviews, 28: 239-259.

20. Sena LM, Bueno C, Pobbe RL et al. (2003). The dorsal raphe nucleus exerts opposed control on generalized anxiety and panicrelated defensive responses in rats. Behavioural Brain Research, 142: 125-133. 